\title{
Atomic-scale study of friction and energy dissipation
}

\author{
S. Ciraci ${ }^{\text {a, } *}$, A. Buldum ${ }^{\text {b }}$ \\ a Department of Physics, Bilkent University, Ankara 06533, Turkey \\ b Department of Physics, The University of Akron, Akron, OH 44325, USA
}

Received 12 July 2002; received in revised form 30 September 2003

\begin{abstract}
This paper presents an analysis of the interaction energy and various forces between two surfaces, and the microscopic study of friction. Atomic-scale simulations of dry sliding friction and boundary lubrication are based on the classical molecular dynamics (CMD) calculations using realistic empirical potentials. The dry sliding of a single metal asperity on an incommensurate substrate surface exhibits a quasi-periodic variation of the lateral force with two different stick-slip stage involving two structural transformation followed by a wear. The contact area of the asperity increases discontinuously with increasing normal force. Xe atoms placed between two atomically flat Ni surfaces screen the Ni-Ni interaction, decrease the corrugation of the potential energy as well as the friction force at submonolayer coverage. We present a phononic model of energy dissipation from an asperity to the substrates.
\end{abstract}

(c) 2003 Elsevier Science B.V. All rights reserved.

Keywords: Atomic-scale study; Friction; Stick-slip

\section{Introduction}

The relative motion of two objects at close proximity (sliding, rolling, or motion in the perpendicular direction) induces non-conservative forces, which in turn, give rise to the loss of mechanical energy by resisting the motion. This phenomenon is called friction and is relevant for various disciplines in science and technology [1-4]. The short- and long-range interactions between two objects are the cause of the friction and energy damping therefrom $[5,6]$. Depending on the distance between objects and also on their relative lateral positions, the magnitude of the interaction potential varies and it can be either attractive or repulsive. The moving objects are either in direct contact through the asperities or lubricants are introduced between them to reduce the friction. The dry sliding friction between atomically flat, commensurate sliding surfaces perhaps is the simplest but most fundamental type of friction in tribology. Depending on the conditions, it may include several interesting phenomena such as adhesion, wetting and atom transfer, bond breaking and bond formation, strain-induced structural transformations and local surface reconstruction, anisotropy in stick-slip motion, and dissipation of non-equilibrium distribution of phonons. Calculations using Tomlinson's model

\footnotetext{
* Corresponding author. Tel.: +90-312-290-1216; fax: +90-312-266-4579.

E-mail address: ciraci@fen.bilkent.edu.tr (S. Ciraci).
}

[7] have indicated also bistability in the stick-slip behavior [8]. In boundary lubrication, foreign atoms, such as Xe atoms at submonolayer coverage, prevent the surfaces of moving objects from making adhesive contact. No matter what the type and scale of the friction, the atomic process between the sliding or moving objects is crucial for friction. During various atomic processes, the energy of motion is damped by phononic and electronic mechanisms.

Recent atomic-scale experimental investigations $[9,10]$ of the interaction between solid surfaces in dry sliding, and between surface and lubricant atoms in boundary lubrication have shed light on the underlying microscopic mechanism of friction. Theoretical studies using atomistic models $[7,8,11,12]$, which were treated by large-scale classical molecular dynamics (CMD) simulations [13-16] and also by the first-principles calculations $[6,17]$ have provided insight for a better understanding.

The atomic-scale analysis of the interaction between sliding surfaces is necessary to understand the non-conservative lateral forces and the mechanism of energy dissipation in friction. It is hoped that a better control of friction will be possible with the knowledge gained from atomic-scale studies. In this paper, we present a concise analysis of atomic-scale friction and energy dissipation based on the results obtained by our earlier studies. In Section 2, we review our earlier ab initio atomic force calculations and discuss the nature of interaction forces between sliding surfaces and lubricant molecules. Important aspects of dry 
sliding friction and boundary lubrication are discussed in Sections 3 and 4, respectively. In Section 5, we present our model of phononic energy dissipation and energy transfer through nanoparticles.

\section{Interactions between surfaces}

Long- and short-range interactions occur between the surfaces or lubricant molecules. The long-range (or Van der Waals) forces, which are weak body forces generated from the dipole-dipole interaction between two electrodes, are normally added to the normal (applied force $F_{\mathrm{N}}$ ) and short-range force. Charging of one of the moving objects can give rise to long-range coulombic forces in dielectric medium. The origin of the short-range (or chemical) forces is the coulombic and exchange potential due to charge redistributions between two surfaces. Its range is only 7-9 from the center of the atoms, and exponentially decays to zero with increasing spacing, $z$. The short-range interaction energy $E_{\mathrm{I}}$, between two substrates (or surfaces) (A and B) is a function of spacing $z$, and can be calculated from the first-principles:

$E_{\mathrm{I}}=E_{\mathrm{T}}[\mathrm{A}+\mathrm{B}]-E_{\mathrm{T}}[\mathrm{A}]-E_{\mathrm{T}}[\mathrm{B}]$,

in terms of the total energies of the $(\mathrm{A}+\mathrm{B}), E_{\mathrm{T}}[\mathrm{A}+\mathrm{B}]$, and those of $\mathrm{A}$ and $\mathrm{B}, E_{\mathrm{T}}[\mathrm{A}]$ and $E_{\mathrm{T}}[\mathrm{B}]$, respectively. The minimum of $E_{\mathrm{I}}(z)$ under zero normal force (at $z=z_{0}$ ) corresponds to the adhesion energy $E_{\mathrm{a}}$. The interaction energy, $E_{\mathrm{I}}$ between two atomically flat and commensurate surface exhibits a periodic variation, if one of the surfaces moves laterally along a direction at constant separation from the other surface. Normally, $E_{\mathrm{I}}(z)$ at a given lateral $(x, y)$ position decays exponentially as $z \rightarrow \infty$, but it becomes positive for small $z$ indicating a repulsive interaction between surfaces. The interaction energy can be parameterized by a Rydberg function, $E_{\mathrm{I}}^{*}\left(z^{*}\right)=-\left(1+z^{*}\right) \exp \left(-z^{*}\right)$ with $E_{\mathrm{I}}^{*}=E_{\mathrm{I}}(z) / E_{\mathrm{a}}$ and $z^{*}=z-z_{0}$. However, $E_{\mathrm{I}}(z)$ displays a complex behavior when the relaxation of atoms become significant at small $z$.

The negative of the first derivative of $E_{\mathrm{I}}$ with respect to the lateral displacement $s=\left(x^{2}+y^{2}\right)^{1 / 2}$, i.e. $-\partial E_{\mathrm{I}} / \partial s$ is the lateral force between two surfaces at constant separation $z=$ $z_{\mathrm{c}}, F_{\mathrm{L}}\left(s, z_{\mathrm{c}}\right)$. The perpendicular and lateral components of the short-range force can also be calculated accurately from the first-principles by using the Hellmann-Feynman theorem. The short-range force along $\alpha$-direction on the atom $i$ is given by

$F_{i, \alpha}=-\left\langle\frac{\Psi(\boldsymbol{r}) \mid \partial \mathcal{H}\left(\tau_{i}\right)}{\partial \tau_{i, \alpha} \mid \Psi(\boldsymbol{r})}\right\rangle$,

where $\tau_{i}$ is the position vector of the atom $i$, and $\Psi$ is the wave function. By an appropriate sum, the force on a surface or on a particular asperity can be calculated. The perpendicular and lateral force variations between two parallel $\mathrm{Al}\left(\begin{array}{lll}1 & 0 & 0\end{array}\right)$ surfaces are calculated from the first-principles [6]

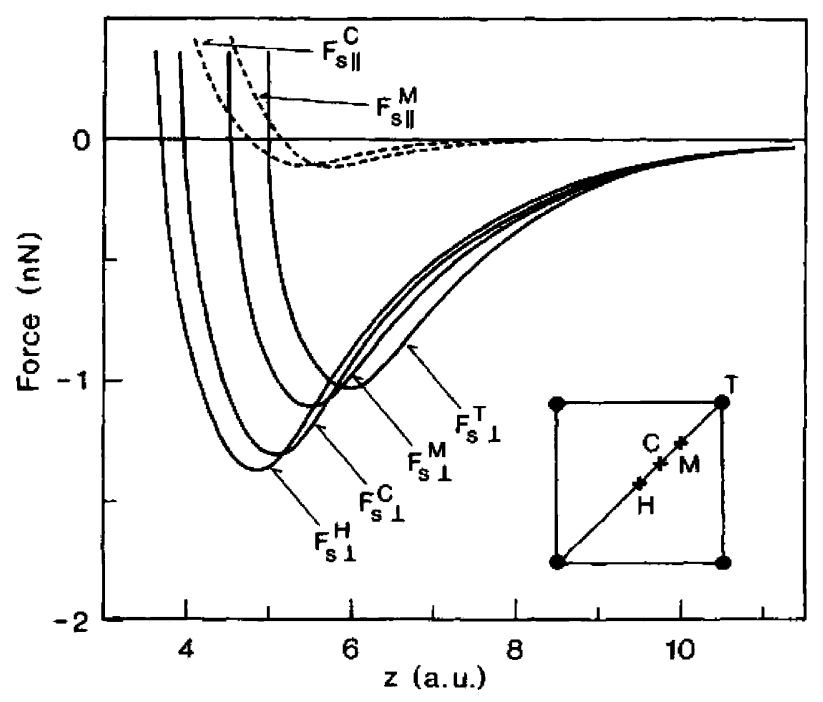

Fig. 1. Short-range perpendicular $F_{\mathrm{S} \perp}$ and lateral $F_{\mathrm{S} \|}\left(F_{\mathrm{L}}\right)$ forces acting on an $\mathrm{Al}$ atom in a (100) layer facing an $\mathrm{Al}(100)$ slab at $\mathrm{H}-, \mathrm{C}-$, $\mathrm{M}$ - and T-sites versus the separation $z$. Forces are calculated using the first principles pseudopotential plane wave method within local density approximation (1 a.u. $=0.529 \AA$ ). Reproduced from [6].

and are illustrated in Fig. 1. Owing to the non-adiabatic changes in the relative motion of $\mathrm{A}$ and $\mathrm{B}$, non-conservative forces are generated, which in turn gives rise to friction. This way, the mechanical energy is transformed to heat energy and is eventually damped away. In the following two sections, large-scale atomic simulations of friction and boundary lubrication will be discussed. Since the first-principle calculations of large systems are time consuming, the atomic simulations were carried out by expressing the interaction between A and B in terms of realistic empirical potentials.

\section{Sliding friction}

The surfaces in relative motion are rarely atomically flat, but they have several asperities. The surfaces come in contact through these asperities. Here, the sliding friction and wear are presented by the atomic simulations of the lateral motion of a Ni asperity over the $\mathrm{Cu}$ surface [15]. The pyramidal asperity is formed from $\mathrm{Ni}\left(\begin{array}{lll}1 & 1 & 1\end{array}\right)$ atomic planes. The substrate has $14 \mathrm{Cu}\left(\begin{array}{lll}1 & 1 & 0\end{array}\right)$ planes which are parallel, but incommensurate to the $\mathrm{Ni}\left(\begin{array}{lll}1 & 1 & 1\end{array}\right)$ planes. The relative motion occurred under the constant normal force $F_{\mathrm{N}}=2.64 \mathrm{nN}$ which is uniformly distributed on the top plane of the asperity. State-of-the-art classical molecular dynamics calculations are performed by using embedded atom potential [18]. The bottom four layers of the $\mathrm{Cu}$ substrate were kept fixed. The top two layers of the asperity are taken robust with fixed atomic arrangement. In the course of lateral motion, these robust layers of the asperity are translated in increments of $\Delta s=0.05 \AA$. At the end of each displacement, all the dynamic atoms of the asperity and substrate (i.e. $120 \mathrm{Ni}$ and 
$1650 \mathrm{Cu}$ atoms) are relaxed to attain their steady state positions in 500 steps. In each time step, $\Delta t=10^{-15} \mathrm{~s}$, the position and velocities of all dynamic atoms are determined by solving classical equation of motion. The temperature is fixed at $4 \mathrm{~K}$ by rescaling the velocities of all dynamic atoms at the end of each step.

Once the contact between substrate and asperity is established under $F_{\mathrm{N}}$, the single apex atom and the subsequent layer of the $\mathrm{Ni}$ asperity disappear. While some $\mathrm{Ni}$ atoms substitute $\mathrm{Cu}$ atoms at the contact, some of them are accumulated behind the asperity. On the other hand, some $\mathrm{Cu}$ atoms migrate to the asperity. In spite of the fact that the cohesive energy of $\mathrm{Ni}$ is higher than that of $\mathrm{Cu}$, two Ni layers adjacent to contact change from the (1 111 ) structure to the (1 110$)$ structure of $\mathrm{Cu}$ substrate. The variation of the lateral force $F_{\mathrm{L}}$ with the displacement, $s$ is shown in Fig. 2. After $N$ increments, the displacement becomes $s=N \Delta s$. By definition, $F_{\mathrm{L}}(s)<0$ resists to the motion. We point out following features in Fig. 2. (i) The abrupt changes of $F_{\mathrm{L}}(s)$ display a quasi-periodic variation; in each period, two features indi-

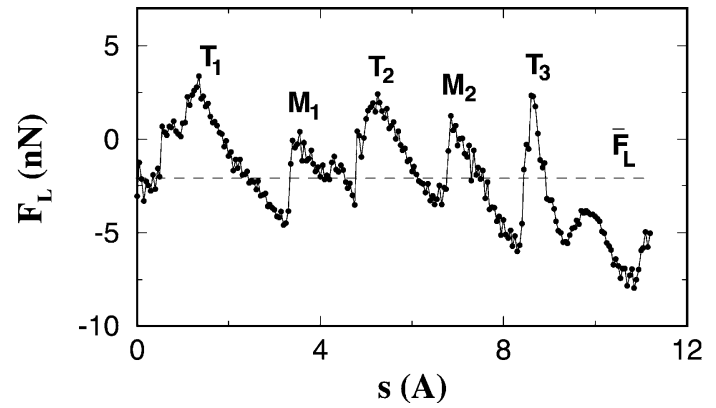

Fig. 2. Quasi-periodic variation of the lateral force $F_{\mathrm{L}}$ with the sliding $s$ of the $\mathrm{Ni}\left(\begin{array}{lll}1 & 1 & 1\end{array}\right)$ asperity on the $\mathrm{Cu}\left(\begin{array}{lll}1 & 1 & 0)\end{array}\right)$ surface. The average lateral force (or friction force) is shown by dashed line. $F_{\mathrm{L}}(s)$ opposing to the motion of the asperity is taken negative. $\mathrm{T}_{n}$ and $\mathrm{M}_{n}$ stand for the different structural transformations in the $n$th period. Reproduced from [15].

cated as $\mathrm{T}$ and $\mathrm{M}$ occur consecutively. (ii) Between $\mathrm{T}_{n}$ and $\mathrm{M}_{n}, F_{\mathrm{L}}(s)$ first decreases and then increases suddenly, and shows a quasi-elastic behavior. The work done against $F_{\mathrm{L}}(s)$ over a period $T_{n}$ to $T_{n+1}$, i.e. $-\int_{T_{n}}^{T_{n+1}} F_{\mathrm{L}}(s) \mathrm{d} s$ changes from
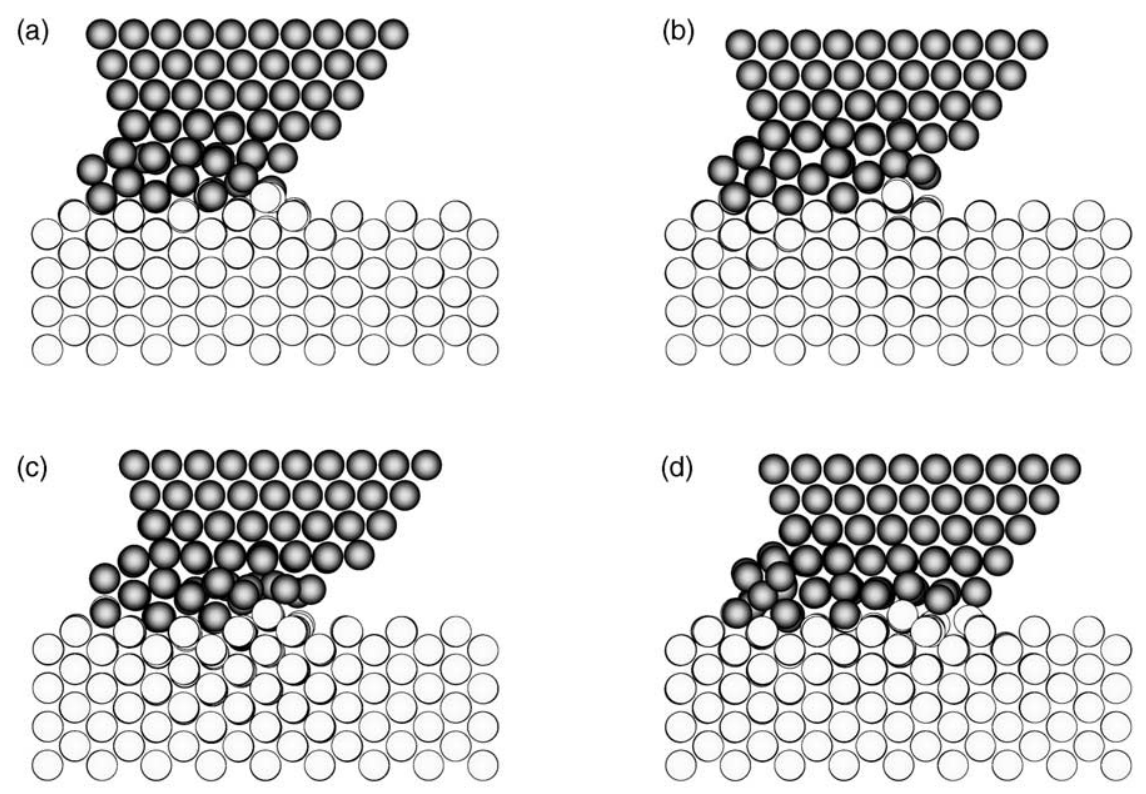

(e)

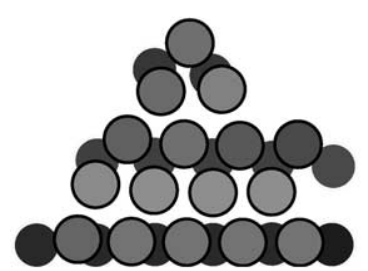

(f)

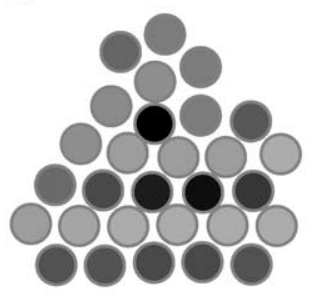

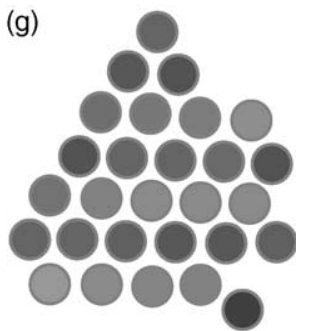

Fig. 3. Snapshots from the side view of atomic structure calculated for the Ni(1 111$)$ asperity (dark gray circles) sliding on the Cu(1 10$)$ surface (light gray circles). Structures before and after $T_{1}$ are shown in (a) and (b); and after and before $M_{1}$ in (c) and (d). Panel (e) shows the $x y$ projection of the atoms in the $\mathrm{Ni}$ layer experiencing a structural transformation. Light gray circles correspond to the fifth $\mathrm{Ni}(111)$ layer from the top before $T_{1}$; after $T_{1}$ this layer has changed to the $\mathrm{Ni}\left(\begin{array}{lll}1 & 1 & 0)\end{array}\right)$ that is commensurate to the $\mathrm{Cu}\left(\begin{array}{lll}1 & 10)\end{array}\right)$ as shown by the dark circles. Panel (f) shows the two $\mathrm{Ni}(110)$ layers corresponding to the situation in (c) before the transformation $M_{1}$. These two layers merged into one $\mathrm{Ni}(111)$ layer in panel (g) as described by the side view (d) after $M_{1}$. Reproduced from [15]. 
period to period. This deviation from the Amonton's law is explained by the growth of the junction in the course of sliding. Quasi-elastic variation of $F_{\mathrm{L}}(s)$ in Fig. 2 is a peculiarity of the stick-slip process in sliding friction. It is also of particular interest that the sliding of a metal asperity on an incommensurate metal surface is shown to yield also a stick-slip behavior. We expect, however, that the stick-slip behavior can be washed out if the sliding involves multiple asperity.

In Fig. 3 snapshots of side and top views of atoms in the layers where the sliding takes place are shown only for $T_{1}$ and $M_{1}$. After $T_{1}$, the lowest $\mathrm{Ni}\left(\begin{array}{lll}1 & 1 & 1\end{array}\right)$ layer which was in registry with the asperity undergoes a structural transformation and becomes matched to the lower Ni layer that was already changed to the lattice structure of $\mathrm{Cu}\left(\begin{array}{lll}1 & 1 & 0\end{array}\right)$. The amount of mechanical energy implemented in this structural transition is calculated to be $\sim 3 \mathrm{eV}$. Beyond any $T_{n}, F_{\mathrm{L}}(s)$ changes linearly and becomes negative so that it resists to the sliding. In this first stick stage, a strain energy of $2.8 \mathrm{eV}$ is stored in the interface. After $\sim 1 \AA$ displacement, the stick stage ends and the slip starts with the second structural transition $M_{1}$, in which two asperity layer commensurate with the substrate merge into a single (1 111$)$ layer of Ni. The transition starts in front of the asperity and ends at its back, and $\left|F_{\mathrm{L}}(s)\right|$ changes abruptly. Here, one layer of asperity disappears and hence the slip ends with a wear. In this process, part of the strain energy is transformed into non-equilibrium distribution of phonons and dissipated in whole system. The incommensurate-commensurate structural transition in each period $T_{n}-M_{n}$, have interesting features [19]. The transition occurs in a short time interval; starting from one ordered 2D structure it ends up in a different ordered atomic structure.

\section{Boundary lubrication}

In studying the boundary lubrication, we consider two $\mathrm{Ni}\left(\begin{array}{lll}1 & 1 & 0\end{array}\right)$ slabs in relative motion. Each slab consists of eight (1 110$)$ atomic planes. Periodic boundary conditions are used in the lateral (or $x y$ ) plane. The lubrication is realized by placing Xe atoms between two slabs at different coverage. The relative motion occurs along the long edge of the (1 110$)$ surface unit cell under the normal load $F_{\mathrm{N}}=0.03 \mathrm{eV} /(\AA$ atom). The empirical potential parameters and other details of the calculation are given elsewhere [16].

In order to understand the effect of lubricant, we first examine the variation of potential energy with the displacement, $V_{\mathrm{T}}(s)$ as a function of coverage $\Theta=0,0.64$ and 0.84 . In the case of $\Theta=0$ (dry sliding), the corrugation between minimum and maximum values of potential energy, $\Delta V_{\mathrm{T}}$ is $170 \mathrm{eV}$, and the structure near the interface becomes disordered before $V_{\mathrm{T}}$ attains its maximum value. The implementation of the Xe atoms between two Ni(1 10$)$ slabs with coverage $0<\Theta<1$ changes the above situation dramatically. $\Delta V_{\mathrm{T}}(s)$ is reduced from $170 \mathrm{eV}$ at $\Theta=0$ to $12.5 \mathrm{eV}$ at $\Theta=0.64$. Xe atoms reduce the interaction between Ni sur- faces and prevent the system from any defect formation under the loading force, except some structural transformation within the lubricant layer. As a result, the average friction force is reduced, and so is the average energy dissipation. The variation of $V_{\mathrm{T}}(s)$ and $F_{\mathrm{L}}(s)$ becomes also regular and periodic in the presence of lubricant Xe atoms. The minimum of the total potential occurs when $\mathrm{Ni}$ atoms at both surfaces face each other (T-site), and Xe atoms are located between the hollow $(\mathrm{H})$ sites of both surface. By translating the upper slab, the $\mathrm{Xe}$ atoms are forced to move in the same direction. This increases $V_{\mathrm{T}}$ until $s<(n-1) a+a / 2$; thereafter, the potential energy is lowered since part of the $\mathrm{Xe}$ atoms raise and move to the $\mathrm{H}$-site of the upper surface, while the rest of them keep their positions at the $\mathrm{H}$-site of the lower surface. Following this stage, $V_{\mathrm{T}}(s)$ starts to decrease and the spacing between two adjacent rows of Xe (each row is associated with different surfaces) starts to open. Upon further displacement, the $\mathrm{H}$-sites of both slabs become in registry again, and the raised Xe's are lowered to the H-sites of the lower surface by leaving one row of vacant $\mathrm{H}$-sites. The complex motion of Xe atoms is reminiscent of the motion of the edge dislocation whereby the lateral force is reduced. Since such a vacant positions, which are necessary for the relocation of $\mathrm{Xe}$ atoms during the sliding are absent at full coverage, $\Delta V_{\mathrm{T}}$ increases from $12 \mathrm{eV}(\Theta=0.64)$ to $20 \mathrm{eV}(\Theta=1)$. In Fig. 4, we show how the Xe atoms are rearranged and how the slabs can slide without any major defect formation.

\section{A microscopic model for energy dissipation}

The dissipation of energy transformed from the mechanical energy to the heat energy is crucial for the friction as well as wear. The dissipation process is rather complex and involves different mechanisms, such as non-equilibrium phonon and electron-hole creation, bond breaking and electron emission, defect formation and structural transformation, and wear. In the systems operating under strong loading force the amount of energy transformed into residual defects can be significant. It is argued that the energy is dissipated mainly by electronic and phononic processes. In the present atomic simulations, a major part of the energy dissipated within the displacement $S, \mathcal{E}_{\mathrm{p}}$, is taken from the system through the thermalization process. The remaining part of the energy $\mathcal{E}_{\mathrm{d}}$ is implemented into the system through the structural transformations induced by the relative motion. If $\mathcal{E}_{\mathrm{d}}$ is negligible, then the "average" dynamic friction constant is obtained from $\mu_{\mathrm{d}}=\mathcal{E}_{\mathrm{p}} /\left(s \times F_{\mathrm{N}}\right)$. Further to this macroscopic approach, it is crucial to understand how the mechanical energy is transformed to phonon energy and how these phonon energies are equilibrated. The dissipation mechanisms are important not only for friction and lubrication, but also for electrical and thermal conduction in nanoscience and molecular electronics. Here, we study the following phononic model. 

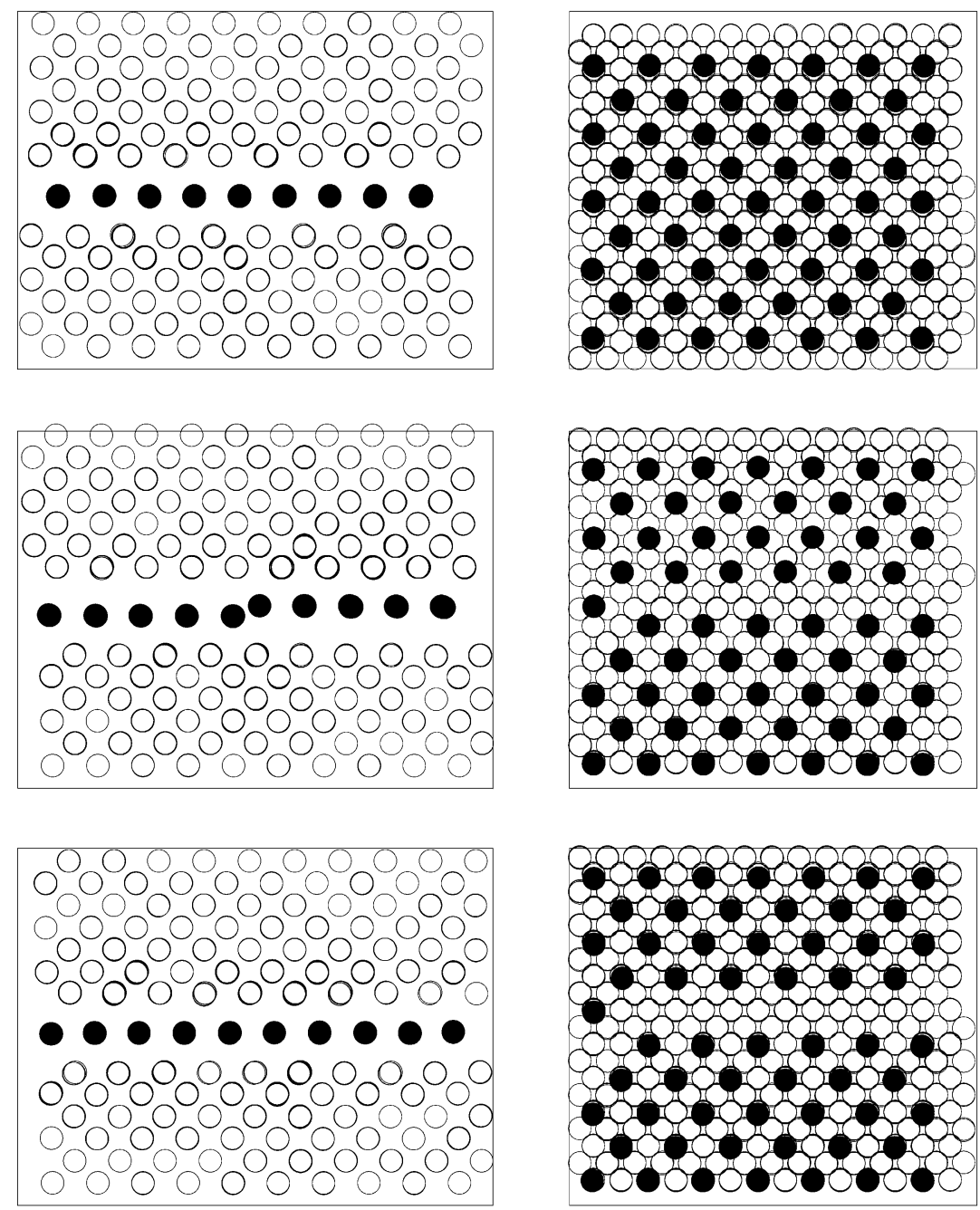

Fig. 4. Snapshots of side and top views of the upper $\mathrm{Ni}(110)$ slab moving laterally above the lower $\mathrm{Ni}(110)$ slab with $\mathrm{Xe}$ coverage $\Theta=0.84$. Upper, middle and lower panels correspond to the different lateral displacements in increasing order. Open and dark circles represent $\mathrm{Ni}$ and $\mathrm{Xe}$ atoms, respectively. Reproduced from [16].

We consider a nanoparticle or a molecule (also asperity) weakly coupled to the surfaces. In the course of relative motion, the elastically deformed state of the nanoparticle, $\boldsymbol{\Psi}_{m}$ depends on the displacements of atoms $\boldsymbol{u}_{i}$. When the deformation is released suddenly, new phonons beyond the thermal equilibrium are generated. Then, the (static) deformed state $\boldsymbol{\Psi}_{m}$ is expressed by the linear combination of the vibrational states [20], which, in turn, can be related to the occupation numbers of the corresponding normal modes, i.e. $\boldsymbol{\Psi}_{m}\left(n_{1}, \ldots n_{q}, \ldots\right)$. This means that the occupation number of certain phonons are increased and hence a non-equilibrium state is created. The number of excited phonons is given by $\Delta n_{q}=n_{q}\left(\Omega_{q}, T\right)-n_{q}^{0}\left(\Omega_{q}, T_{0}\right)$ as a difference between non-equilibrium and thermal equilibrium distribution. Here, $\Omega_{q}$ is the frequency of the particular phonon mode, and $T$ and $T_{0}$ are temperatures $\left(T>T_{0}\right)$ corresponding to non-equilibrium and thermal equilibrium distributions, respectively. Then, the amount of energy transformed to a particular phonon energy (or heat energy) is given by: $\delta V_{q}=\hbar \Omega_{q} \Delta n_{q}$,

Then, the total mechanical energy which is transformed to the vibrational energy is $\delta V_{\mathrm{T}}=\sum_{q} \delta V_{q}$ is dissipated according to the expression:

$\delta V_{\mathrm{T}}(t)=\sum_{q} \delta V_{q}(t=0) \exp \left[-R\left(\Omega_{q}\right) t\right]$.

The value of $R\left(\Omega_{q}\right)$, which is related to the decay rate of a particular mode $\Gamma_{q}$ by $R_{q}\left(\Omega_{q}\right)=\Gamma_{q} n_{q}\left(\Omega_{q}, T\right)$, depends on the material parameters of the surfaces, (such as the velocity of sound of the slab $v_{j}$, the energy of the particular mode, $\epsilon_{q}=\hbar \Omega_{q}$, and the phonon density of states of the substrates, $\left.D\left(\Omega_{k}\right)\right)$ is expressed by Buldum et al. [21-24]. They performed a study of energy dissipation from a nanoparticle consisting of $14 \mathrm{Cu}$ atoms to the $\mathrm{Cu}$ surfaces by using the above formalism. They found that the lowest energy mode, $\epsilon_{q=1}=\hbar \Omega_{q=1}=89.7 \mathrm{~K}$, has the decay rate, $\Gamma_{q=1}=R\left(\Omega_{q=1}\right) n_{q=1}=0.41 \times 10^{11} \mathrm{~s}^{-1}$ and higher frequency modes decay faster. 


\section{Conclusions}

In this work, we present important features of dry sliding friction and boundary lubrication obtained from extensive atomic-scale classical molecular dynamics simulations. For the dry sliding friction, we treated the lateral motion of an asperity in contact with an atomically flat substrate surface. Following features are emphasized for an asperity incommensurate with the substrate surface: (i) The contact area increases with increasing normal force in discrete steps. (ii) The lateral force exhibits stick-slip behavior. (iii) While one layer of asperity changes and matches the substrate lattice in the first slip, two asperity layers merge into one through structural transformation during the second slip. This leads to wear. (iv) The sliding of a flat $\mathrm{Ni}(100)$ on he pseudomor-

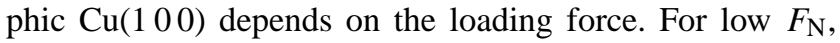
$F_{\mathrm{L}}(s)$ displays quasi-linear variation in the stick stage. At high $F_{\mathrm{N}}$, the stick-slip motion becomes less regular.

The analysis of the dynamics of $\mathrm{Xe}$ atoms as lubricant between two lattice matched $\mathrm{Ni}(110)$ surface in relative motion revealed how a lubricant operates. Xe atoms screen the interaction between two $\mathrm{Ni}$ surfaces and prevents the defect formation or structural deformation. The corrugation of the total energy is reduced dramatically as the coverage of Xe increases.

Finally, we discuss a phononic model of energy dissipation from an asperity to the moving slabs. High frequency non-equilibrium modes are found to decay faster than lower frequency modes.

\section{Acknowledgements}

This work was partially supported by TÜBA Academy of Science of Turkey.

\section{References}

[1] F.P. Bowden, D. Tabor, Friction and Lubrication, Methuen, London, 1965.

[2] E. Rabinowicz, Friction and Wear, Wiley, New York, 1965.

[3] M. Grunze, H.J. Kreuzer, Adhesion and friction, in: Springer Series in Surface Science, vol. 17, Springer, Berlin, 1989.

[4] Physics of sliding friction, in: B.N.J. Persson, E. Tosatti (Eds.), Nato Advanced Study Institute Series E: Applied Science, vol. 311, Kluwer Academic Publishers, Dordrecht, 1966.

[5] J.N. Israelachvili, Intermolecular and Surface Forces, Academic Press, London, 1985

[6] S. Ciraci, E. Tekman, A. Baratoff, I.P. Batra, Phys. Rev. B 46 (1992) 10411.

[7] G.A. Tomlinson, Philos. Mag. 7 (1929) 905.

[8] A. Buldum, S. Ciraci, Phys. Rev. B 55 (1997) 2606.

[9] C.M. Mate, G.M. McClelland, R. Erlandsson, S. Chiang, Phys. Rev. Lett. 59 (1987) 1942.

[10] E. Meyer, R. Overney, D. Brodbeck, L. Howald, R. Lüth, J. Frommer, H.J. Güntherodt, Phys. Rev. Lett. 69 (1992) 1777.

[11] J. Frenkel, T. Kontorova, Phys. Z. Sowjet 13 (1938) 1.

[12] B.N.J. Persson, Phys. Rev. Lett. 71 (1993) 1212; B.N.J. Persson, Phys. Rev. B 50 (1994) 4771.

[13] U. Landman, W.D. Luedtke, N.A. Burnhan, R.J. Colton, Science 248 (1990) 454

[14] P.A. Thomson, M.O. Robbins, Science 250 (1990) 792.

[15] A. Buldum, S. Ciraci, I.P. Batra, Phys. Rev. B 57 (1998) 2468.

[16] A. Buldum, S. Ciraci, Phys. Rev. B 60 (1999) 1982.

[17] D. Tomanek, W. Zhong, H. Thomas, Europhys. Lett. 15 (1991) 887.

[18] M.S. Daw, M.I. Baskes, Phys. Rev. B 29 (1984) 6443; S.M. Foiles, M.I. Baskes, M.S. Daw, Phys. Rev. B 33 (1986) 7983.

[19] A. Buldum, S. Ciraci, Phys. Rev. B 55 (1997) 12892.

[20] G.P. Srivastava, The Physics of Phonons, Adam Hilger, Bristol, 1990.

[21] A. Buldum, D.M. Leitner, S. Ciraci, Phys. Rev. B 59 (1999) 16042.

[22] A. Buldum, D.M. Leitner, S. Ciraci, Europhys. Lett. 47 (1999) 208.

[23] A. Buldum, S. Ciraci, C.Y. Fong, J. Phys.: Condens. Matter 12 (2000) 3349.

[24] A. Ozpineci, S. Ciraci, Phys. Rev. B 63 (2001) 125415. 